\title{
Bone inflamm-ageing
}

Bone regeneration and fracture repair mechanisms are compromised as people age. One mechanism to account for this decline is that skeletal stem and progenitor cells (SSPCs) are more prone to differentiate into fat-producing adipocytes than into bone-producing osteoblasts. New research indicates that this switch in SSPC differentiation occurs as a result of chronic inflammation that develops as we age.

"In the elderly, an imbalance between adaptive and innate immunity, as well as a progressive accumulation of senescent cells, lead to elevated levels of pro-inflammatory mediators, a process known as 'inflamm-ageing"', says Philipp Leucht, corresponding author of the new research.

Leucht and his team took bone samples from patients undergoing fracture fixation surgery (see image) and found an association between low SSPC number and a slow time to fracture union. This finding was replicated in a mouse tibial monocortical defect model, with better bone regeneration and a greater number of SSPCs in young ( 12 weeks) mice than in middle-aged (52 weeks) mice.

To distinguish chronological ageing from inflamm-ageing, the researchers showed that young $\mathrm{N}_{\mathrm{kb}} \mathrm{1}^{-/-}$mice, which have constitutively active NFKB signalling and are a model of modest chronic inflammation, also have a low number of SSPCs. Furthermore, SSPCs from these mice display a senescent phenotype, an increased ability to differentiate into adipocytes and a reduced capacity for chondrogenic and osteogenic differentiation.

The researchers showed that treating middle-aged (52 weeks) mice with NSAIDs resulted in an increase in the number of SSPCs, and the transcriptional signature and function of these cells became more osteogenic, resembling SSPCs from young mice.

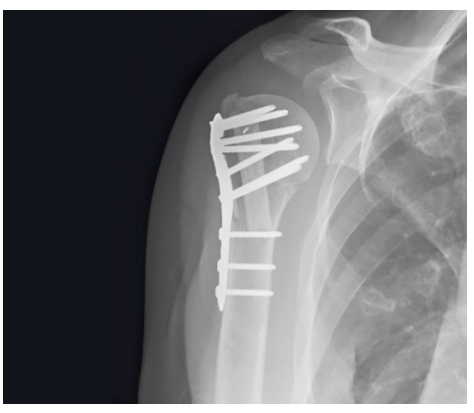

X-ray image of right shoulder with proximal humerus fracture after operative fixation. Image courtesy of P. Leucht, New York University School of Medicine, USA.

"Anti-inflammatory drugs could be taken prior to elective orthopedic surgery to improve stem cell number and function," hypothesizes Leucht "But further research should be to target the SSPCs, to ameliorate the effects of age-associated inflammation on this cell type without inhibiting inflammation that is necessary for successful bone regeneration."

Nicholas J. Bernard

an increase in the number of SSPCS

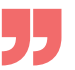

\section{Blocking CD40-CD40L interactions in Sjögren syndrome}

Ectopic lymphoid structures (ELSs) can form in the salivary glands of patients with Sjögren syndrome, and are thought to contribute to pathology. Previous studies have implicated the CD40-CD40 ligand (CD40L) pathway in ELS formation and function. In a new study from a group at the Novartis Institutes for Biomedical Research, therapeutic blockade of CD40L ameliorated disease in a non-obese diabetic (NOD) mouse model of secondary Sjögren syndrome.

"We found evidence of chronic CD40 expression and pathway activation in the salivary glands of these mice, indicating that signalling downstream of this receptor could have a function in Sjögren syndrome disease progression," states corresponding author James Rush.

The researchers treated the mice biweekly with an anti-CD40L antibody (MR1) from 12 weeks of age, at which stage the mice develop focal cellular infiltrates. The treatment inhibited sialadenitis, reduced the number of infiltrating lymphocytes and macrophages, as well as the number of ELSs, and suppressed autoantibody production in these mice.

By contrast, the treatment had no effect on the expression of the

The treatment inhibited sialadenitis ... and suppressed autoantibody production

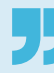
water channel protein AQP5 in the salivary glands. This protein is involved in regulating saliva and tear production but whether it is a useful surrogate marker of salivary gland function in Sjögren syndrome is unclear.

"Collectively, our data suggest that therapeutic blockade of the CD40-CD40L costimulatory pathway

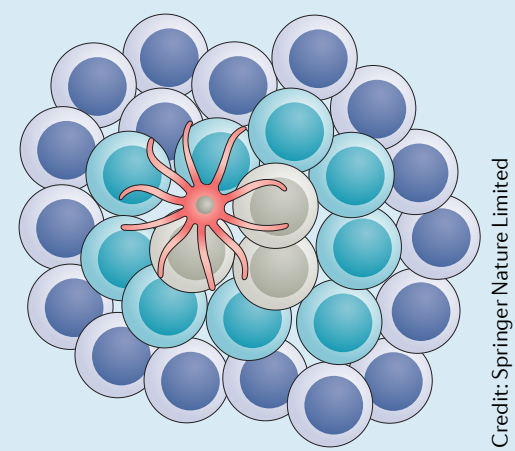

could provide a beneficial therapeutic effect in patients with Sjögren syndrome, a notion confirmed in a recent Phase II study clinical study of the anti-CD40 monoclonal antibody Iscalimab (CFZ533)," says Rush. "Future work could focus on how CD40 blockade affects salivary gland function, as well as whether chronic therapeutic dosing is required for a disease modifying effect." Jessica McHugh

ORIGINAL ARTICLE Wieczorek, G. et al. Blockade of CD40-CD154 pathway interactions suppresses ectopic lymphoid structures and inhibits pathology in the NOD/ShiLt] mouse model of Sjögren's syndrome. Ann. Rheum. Dis. https://doi. org/10.1136/annrheumdis-2018-213929 (2019) 